\title{
Diacronie
}

Studi di Storia Contemporanea

$N^{\circ} 23,3$ | 2015

Mediterraneo cosmopolita

\section{Elisa Grandi, Deborah Paci (a cura di), La politica degli esperti. Tecnici e tecnocrati in età contemporanea}

\section{Roberto Ventresca}

\section{Q OpenEdition}

Edizione digitale

URL: http://journals.openedition.org/diacronie/2406

DOI: 10.4000/diacronie.2406

ISSN: 2038-0925

Editore

Association culturelle Diacronie

Notizia bibliografica digitale

Roberto Ventresca, «Elisa Grandi, Deborah Paci (a cura di), La politica degli esperti. Tecnici e tecnocrati in età contemporanea », Diacronie [Online], $N^{\circ} 23,3$ | 2015, documento 7, Messo online il 29 octobre 2015, consultato il 23 septembre 2020. URL : http://journals.openedition.org/diacronie/2406 ; DOI :

https://doi.org/10.4000/diacronie.2406 
N. 23 | 3|2015 Mediterraneo cosmopolita: le relazioni culturali tra Turchia ed Europa

\title{
RECENSIONE:
}

\section{Elisa GRANDI, Deborah PACI (a cura di), La politica degli esperti. Tecnici e tecnocrati in età contemporanea, Milano, Edizioni Unicopli, 2014, 264 pp.}

\author{
a cura di Roberto VENTRESCA *
}

Ragionare intorno al complesso intreccio tra la sfera della "tecnica" e quella della "politica" - interpretando la prima come un insieme di competenze e di saperi specifici e socialmente legittimati, e leggendo invece la seconda come lo spazio entro cui si dispiegano le dinamiche del comando, del potere e della resistenza che di rimando vi si oppone - equivale a interrogarsi sulle modalità attraverso le quali le società (e non soltanto quelle occidentali) nel corso degli ultimi secoli hanno governato il proprio rapporto con la modernità, e soprattutto con uno dei suoi "agenti" più significativi, vale a dire lo Stato, insieme con le sue molteplici articolazioni interne (come ad esempio gli organismi della Pubblica Amministrazione). Inoltre, porre l'accento sui percorsi biografici di alcune personalità annoverate nella variegata compagine delle élites tecnocratiche dei secoli XIX-XXI così come avviene nel volume curato da Elisa Grandi e Deborah Paci La politica degli esperti. Tecnici e tecnocrati in età contemporanea significa indagare in sede storiografica il concreto manifestarsi dell'incontro (spesso problematico) tra il mondo dell'expertise tecnica e quello della decisione politica: un tema sul quale si stanno moltiplicando negli ultimi anni numerosissime riflessioni di carattere anche non strettamente storiografico ${ }^{1}$.

\footnotetext{
${ }^{1}$ Si vedano ad esempio FUMIAN, Carlo, «Per una storia della tecnocrazia: utopie meccaniche e ingegneria sociale tra Otto e Novecento", in Rivista storica italiana, CXIII, 3/2012, pp. 908959; RICCIARDI, Ferruccio, Les techniques managériales à l'heure de la guerre froide: la parabole des "relations humaines" dans l'Italie des années 1950, in GONZALEZ BERNALDO, Pilar, HILAIRE-PERÉZ, Liliane (dir.), Les savoirs-mondes. Mobilités et circulation des saviors depuis le MoyenAge, Rennes, Presses universitaires de Rennes, 2015, pp. 287-300; HABERMAS, Jürgen, Nella spirale tecnocratica. Un'arringa per la solidarietà europea, Roma-
} 
Il volume presenta un ventaglio di contributi molto ampio, sia dal punto di vista dei temi analizzati che da quello dei riferimenti cronologici e geografici prescelti: le tre sezioni che compongono il testo - intitolate Le élites tecniche e statali; Esperti economici e pianificazione statale; Il governo dei tecnocrati, a cui si aggiunge un capitolo intitolato Bussole, dove vengono fornite brevi riflessioni su alcuni concetti chiave affrontati nel libro, come Tecnocrazia o Pianificazione Economica - accolgono una serie di saggi che spaziano dalla statistica del lavoro nell'Italia giolittiana alle esperienze tecnocratiche nel Giappone del post-1945; dalla ricostruzione della parabola biografica dell'ingegnere-tecnocrate Henri Fayol (1841-1925) al manifestarsi dell'“utopia cibernetica” in Unione Sovietica tra gli anni Cinquanta e Settanta. Questo brevissimo excursus sulle tematiche affrontate nel volume consente di situare in maniera più approfondita la prospettiva mediante la quale Elisa Grandi e Deborah Paci - storica dell'economia e dei réseaux sociaux presso l'Università di Parigi 7 la prima, esperta di storia culturale e ricercatrice all'Università Ca' Foscari di Venezia la seconda - raccolgono e coordinano i saggi presenti nel libro: una prospettiva, esplicitano le curatrici nell'introduzione, di carattere anzitutto transnazionale, così come viene testimoniato dalla semplice lettura dell'indice (i singoli capitoli analizzano diversi fenomeni tecnocratici relativi alla realtà italiana, belga, francese, argentina, irlandese, giapponese, statunitense, cinese e sovietica); e, in secondo luogo, trans-disciplinare, proprio perché l'ottica metodologica adottata, pur favorendo un approccio di carattere prevalentemente storiografico, si serve degli strumenti analitici propri delle scienze sociali (sociologia, politologia, economia), arricchendo dunque il volume di un valore euristico molto pregnante. Proprio l'introduzione, infatti, condensa al suo interno un insieme di percorsi analitici e di indicazioni bibliografiche (supportate da una più ampia bibliografia finale) molto utili per guidare il lettore tra gli studi più aggiornati sul rapporto tra tecnici, tecnocrati e politici in età contemporanea, in primo luogo attraverso la ricostruzione della genesi storiografica e teorica del concetto di tecnocrazia - a partire dall'uscita del libro di J. Burnham The Managerial Revolution ${ }^{2}$ nel 1941 e dalla sua successiva ricezione in ambito francese - e, in secondo luogo, mediante l'esplicitazione delle domande di fondo che animano la ricerca storica su queste specifiche tematiche: come interpretare e problematizzare il nesso istituitosi tra

Bari, Laterza, 2014 [Ed. originale Im SogderTechnokratie. KleinePolitischeSchriften XII, Berlin, SuhrkampVerlag, 2013]; EASTERLY, William, La tirannia degli esperti. Economisti, dittatori e diritti negati dei poveri, Roma-Bari, Laterza, 2015 [Ed. originale The Tyranny of Experts. Economists, Dictators, and the Forgotten Rights of the Poor, New York, Basic Books, 2013].

${ }^{2}$ BURNHAM, James, La rivoluzione manageriale, Torino, Bollati Boringhieri, 1992 [Ed. originale The ManagerialRevolution. What is happening in the World, New York, The John Day Comp., 1941]. 
expertise tecnica e politica; in che modo si configurano lo spessore decisionale e l'autonomia dei tecnocrati all'interno delle strutture politico-burocratiche entro le quali sono inseriti; quali differenze sussistono tra questa élite delle competenze e l'élite dei politici. Alla luce di questi interrogativi, le curatrici tracciano un profilo interpretativo dell'ampio dibattito sul tema della tecnocrazia in ambito storiografico, passando poi, sempre nell'introduzione, a illustrare sinteticamente il contenuto e le prospettive analitiche dei singoli saggi, che però in questa sede verranno ripercorsi in maniera non sistematica, bensì - per così dire - "tematica", con l'obiettivo di sottolineare i temi ricorrenti e i nuclei argomentativi fondamentali su cui si costruiscono buona parte dei contributi interni al volume.

Una prima osservazione concerne la distinzione terminologica che sussiste tra $\mathrm{i}$ ruolo dei "tecnici" e quello dei "tecnocrati": molto opportunamente le curatrici sottolineano, sulla scorta delle osservazioni di Jean Meynaud, come «i tecnici [siano] agenti che non vanno oltre i limiti del carattere artificiale e subalterno delle tecniche da loro esercitate. I tecnocrati, al contrario, approfittano della tendenza delle tecniche per rendersi indipendenti e valorizzarsi»3. Un passaggio, quest'ultimo, che potrebbe essere reso in maniera ancora più esplicita: l'evoluzione (o, se si vuole, l'involuzione) dalla tecnica in tecnocrazia si produce quando ai saperi e alle competenze tecniche viene conferito uno sbocco propriamente politico, e cioè quando i tecnici agiscono affinché le proprie conoscenze si trasformino in materia di indirizzo politico e governativo all'interno delle società nelle quali essi stessi vivono. Ciò consente di individuare nelle strutture burocratiche dello Stato moderno, nonché nella temperie storica dell'Illuminismo e della Rivoluzione francese, il "luogo" storico in cui emergono in maniera evidente le figure dei tecnici-esperti, chiamati a offrire il proprio bagaglio di saperi al servizio della regolamentazione (e della gouvernamentalité foucaultiana) di tutti quei settori della vita associata - industria, agricoltura, sanità, istruzione rispetto ai quali lo Stato è chiamato ad interessarsi. Forti del riconoscimento sociale di cui essi godono alla luce del proprio status professionale di "esperti" - e cioè portatori di un sapere legalmente, culturalmente e socialmente non solo codificato, ma soprattutto legittimo - i tecnocrati ricoprono un ruolo estremamente significativo nelle dinamiche politiche della società contemporanea, così come i casi di studio analizzati nel volume testimoniano ampiamente.

Non a caso, infatti, uno dei Leitmotiv dei saggi presenti nel libro consiste nel riconoscimento di quanto i tecnocrati interpretino il loro bagaglio di conoscenze

${ }^{3}$ MEYNAUD, Jean, Tecnocrazia e politica, Bologna, Cappelli, 1960, p. 36. La citazione è riportata in GRANDI, Elisa, PACI, Deborah (a cura di), La politica degli esperti. Tecnici e tecnocrati in età contemporanea, Milano, Edizioni Unicopli, 2014, p. 9. 
tecniche alla stregua di una sorta di certificazione di legittimità del proprio operato e delle proprie decisioni, sia che queste ultime si dispieghino nell'ambito di strutture amministrative non direttamente politiche (esemplare la vicenda, analizzata nel saggio di LucRojas, dell'ingegnere Henri Fayol, il quale, nelle pagine del suo Administration industrielle et générale del $1916^{4}$, teorizza - sulla scorta delle sua diretta esperienza professionale e della sua «visione meccanicistica del corpo sociale» - un modello "scientifizzato" di gestione d'impresa), sia che tali decisioni maturino in ambiti governativi e statuali. Leggendo ad esempio le pagine del saggio di Jimena Caravaca, incentrato sull'esperienza economica del governo golpista argentino negli anni Trenta, emerge con forza la profonda convinzione di alcuni tecnocrati economisti (come Raúl Prebisch, il quale, nonostante le sue simpatie socialiste, lavora per il governo golpista: la capacità di "tagliare" trasversalmente lo spettro delle appartenenze politiche costituisce una costante nelle esperienze biografiche di molte figure tecnocratiche) in merito alla "adattabilità" della scienza economica a qualsivoglia orizzonte politico. Proprio in quanto (presunta) "scienza" (e pertanto indubitabilmente vera, dunque neutra), secondo i tecnocrati argentini l'economia potrebbe offrire ricette anti-crisi universalmente valide e politicamente trasversali, sempre che le decisioni in materia di produzione industriale, politica monetaria o misure fiscali vengano decise soltanto da un ristretto gruppo di "esperti" - gli economisti - amministratori di un sapere troppo "complesso" per poter essere compreso (e conteso) dal ceto politico parlamentare. Queste dinamiche prefigurano uno scenario ricorrente all'interno della cosiddetta autonarrazione dei tecnocrati: riservare ai tecnocrati (e ciò avviene sia nei Paesi cosiddetti democratici che nei Paesi autoritari, come nel caso analizzato dalla Caravaca) la gestione esclusiva delle decisioni tecnico-economiche statali comporta la sottrazione delle finalità ultime della politica economica di un Paese alla più ampia discussione democratica, determinando un tal modo una estrema divaricazione tra il concreto esercizio della sovranità popolare e la reale configurazione delle scelte e delle decisioni adottate di volta in volta dai singoli governi.

Questa trasfigurazione della pratica democratica, imposta in nome della presunta neutralità e dell'intrinseca veridicità della scienza economica, non rappresenta però l'esito unico e inevitabile dell'intreccio tra tecnica e politica, come dimostra ad esempio il caso di Giovanni Montemartini (1867-1913), sul quale si focalizza il saggio di Manfredi Alberti: statistico del lavoro impegnato sia nel "laboratorio" della Società

${ }^{4}$ FAYOL, Henri, Direzione industriale e generale. Programmazione, organizzazione, comando, coordinamento, controllo, Milano, Franco Angeli, 1973 [Ed. originale Administration industrielle et générale. Prévoyance, organisation, commandement, coordination, contrôle, Paris, Dunod et Pinat, 1917]. 
Umanitaria di Milano, sia nell'Ufficio del Lavoro del Ministero dell'Agricoltura, Industria e Commercio nel primo decennio del Novecento, Montemartini si adopera affinché il proprio sapere (si tratta infatti di un economista marginalista: una connotazione intellettuale da tenere in considerazione) sulle dinamiche del mondo del lavoro fungano da strumento di conoscenza da mettere al servizio della politica, consentendo a quest'ultima di programmare il proprio intervento nel mercato occupazionale in termini migliorativi per le condizioni dei lavoratori. Proprio ai lavoratori, inoltre, Montemartini si rivolge per ottenere una serie di contributi - in termini di informazione, dati, esperienze - che possano arricchire e integrare le sue analisi statistiche sulla disoccupazione: un esempio, quest'ultimo, di quanto il sapere tecnocratico non si sia sempre configurato, da un punto di vista storico, come una semplice trasmissione di prescrizioni autoritative imposte dall'alto, nonostante si possa presumere come la raccolta e l'utilizzo delle informazioni provenienti "dal basso" restino comunque appannaggio di un certo numero di esperti. Ancora una volta, esulando però dal caso specifico di Montemartini, è sul piano dell'amministrazione "oligarchica" del sapere e delle informazioni (spesso di natura tecnico-economica) che si sostanzia lo status professionale, intellettuale e politico dei tecnocrati.

Tuttavia, articolando ulteriormente una riflessione sul potenziale politico del trattamento delle informazioni da parte di figure tecnocratiche, occorre prestare attenzione alle implicazioni - e alle degenerazioni - insite nella gestione dei flussi di dati ad opera di esperti del settore, proprio in quanto i saperi e le informazioni, anche se osservati nella loro veste puramente quantitativa, risultano essere storicamente e politicamente situati. Prendendo spunto dal contributo di Slava Gerovitch sulla diffusione della cyburocrazia(definita come «un apparato burocratico fornito delle metodologie e della tecnologia cibernetica, computer inclusi»5) nell’Unione Sovietica degli anni Cinquanta-Settanta, si può notare come la produzione di un numero esorbitante di dati, statistiche e documenti elaborati per offrire un supporto informativo alle politiche di intervento economico dei governi in carica possa generare - come di fatto avviene in URSS - un effetto contrario a quello immaginato, e cioè una superfetazione incontrollabile e inutilizzabile di nozioni, la cui immediata conseguenza consiste dunque nella paralisi decisionale. Se in questo specifico case study una parte rilevante della "eterogenesi dei fini” connessa all'eccessiva produzione di informazioni viene svolta dal "sabotaggio" operato dai quadri medio-bassi della pubblica amministrazione sovietica, timorosi di perdere - per via delle innovazioni tecnologiche

5 GEROVITCH, Slava, Cyberocrazia o cyburocrazia? Il destino di un'utopia cibernetica nell'Unione Sovietica, in GRANDI, Elisa, PACI, Deborah (a cura di), op. cit., p. 199. 
imposte dai vertici del Partito comunista - una quota consistente della propria capacità di incidenza e di discrezionalità sulle informazioni che essi amministrano, è interessante notare come le esperienze tecnocratiche descritte nei saggi di cui si compone il volume evidenzino caratterizzazioni politiche estremamente differenti: in alcuni casi esplicitamente reazionarie e autoritarie (si veda il saggio Antonio Cañellas su Laureano López Rodó e sul progetto di riforma tecnocratica dell'Amministrazione statale sotto il regime franchista), in altri apparentemente neutrali o, addirittura, con coloriture "democratiche" (come avviene nell'esperienza dei tecnocrati della Nuova Frontiera kennediana di cui parla Kasper GrotleRasmussen, il quale però insiste molto, forse peccando in scarsa problematizzazione concettuale, sulle capacità "intellettuali” e sulle doti di "razionalità" di questi personaggi, quasi che "ragione" e "razionalità" siano attributi neutrali e oggettivi). Le pagine di questo libro illustrano chiaramente quanto i tecnocrati, nella loro concreta esperienza biografica, badino molto poco alle etichette ideologiche e molto più agli aspetti pragmatici del proprio coinvolgimento politicoamministrativo.

Occorre dunque considerare il fenomeno tecnocratico, quale che sia la sua ispirazione politica o ideologica, come una sorta di destino ineluttabile delle società contemporanee? A questo proposito, qualche lume ci viene offerto dal penultimo saggio del volume, dove Jungwoon Yoon analizza il problema tecnocratico prendendo in esame le caratteristiche della nomenklatura a capo del Partito comunista della Repubblica Popolare cinese. Scorrendo i dati relativi ai rappresentanti del Comitato Centrale e del Politburo che si sono succeduti dalla morte di Mao fino al Congresso del Partito svoltosi nel 2012, Yoon rileva come la composizione sociale e professionale dei membri più importanti del PCC sia variata di molto negli ultimi quarant'anni: se sotto la direzione di Deng Xiaoping, agli inizi degli anni Ottanta, il numero di ingegneri e scienziati naturali risultava preponderante (in quanto il Partito considerava lo sviluppo economico, e soprattutto industriale, una priorità imprescindibile del "nuovo corso" cinese), con l'uscita di scena di Jiang Zemin - anch'egli espressione dell'élite tecnocratica cinese - e l'avvento al potere di Hu Jintao nel 2002 si è via via moltiplicato il numero di alti dirigenti comunisti provenienti perlopiù dal settore finanziario e bancario, nonché da esperienze di ricerca e insegnamento universitario nel campo delle scienze sociali. Questa metamorfosi della classe dirigente cinese, nota Yoon, potrebbe essere messa in relazione con l'emersione di nuove esigenze e nuovi obiettivi nelle politiche del gigante asiatico, come ad esempio una maggiore attenzione alle conseguenze sociali e ambientali dell'impetuoso - e per certi versi "squilibrato" sviluppo economico vissuto dal Paese nel corso degli ultimi anni. 
Queste osservazioni consentono dunque di terminare la nostra analisi con un'ulteriore riflessione: la pervasività del fenomeno tecnocratico all'interno delle dinamiche sociali, politiche e culturali delle società contemporanee costituisce un dato di fatto innegabile. Altrettanto innegabile appare però l'eterogeneità e la "polifonia" di cui si nutre questo stesso fenomeno, il quale, in epoche e contesti storici differenti, acquista una fisionomia sempre rinnovata e originale, proprio perché originali sono i percorsi biografici degli esponenti di queste élites delle competenze (la cui composizione di genere, va notato, è quasi del tutto di carattere maschile). Tuttavia, come sembra emergere dal volume curato da Elisa Grandi e da Deborah Paci, l'analisi dell'intreccio che si è storicamente istituito tra la sfera della politica e quella della tecnica - di cui si parlava all'inizio - non consente letture unidirezionali o men che meno "apocalittiche" (magari ispirate a una presunta vittoria dei "saperi oggettivi" sulla "relatività" del conflitto politico) del fenomeno tecnocratico: a nostro avviso, rimane ancora valida un'acuta osservazione di Alfredo Salsano, il quale si domandava come mai «il trionfo del politico [...] abbia potuto essere presentato come il suo contrario. ì accaduto in realtà che l'integrazione della ragione e del personale tecnici ha fornito al dominio politico, comunque costituito, i mezzi per presentarsi con quei requisiti di legittimazione razionale che gli consentono di presentarsi come pura (e totale) amministrazione» ${ }^{6}$.Ecco: riconoscere l’intrinseca e contraddittoria politicità della pratica tecnocratica, sebbene essa venga declinata in forme e termini spesso estranei alle più classiche dicotomie del politico (quali progresso/conservazione, o autoritarismo/emancipazione), rappresenta un imprescindibile grimaldello concettuale di cui servirsi nell'analisi dei fenomeni storici della contemporaneità.

6 SALSANO, Alfredo, Ingegneri e politici. Dalla razionalizzazione alla «rivoluzione manageriale», Torino, Einaudi, 1987, p. XI. 


\section{* L'autore}

Roberto Ventresca è dottorando presso la Scuola superiore di Studi storici e storico-religiosi (Università di Padova, Università di Verona, Università Venezia - Ca' Foscari), dove sta concludendo una tesi sul ruolo dell'Italia nell'OECE durante la prima legislatura repubblicana (1947-1953). Si è laureato in Storia contemporanea presso l'Università di Bologna e in cotutela presso l'Université Paris 7 - Denis Diderot. Nell'autunno 2014 è stato Visiting Research Student presso la London School of Economics and Political Science.

URL: < http://www.studistorici.com/progett/autori/\#Ventresca >

\section{Per citare questo articolo:}

VENTRESCA, Roberto, «Recensione: Elisa GRANDI, Deborah PACI (a cura di), La politica degli esperti. Tecnici e tecnocrati in età contemporanea, Milano, Edizioni Unicopli, 2014, 264 pp.», Diacronie. Studi di Storia Contemporanea : Mediterraneo cosmopolita: le relazioni culturali tra Turchia ed Europa, 29/10/2015,

URL:< http://www.studistorici.com/2015/10/29/ventresca_numero_23/ >

\section{Diacronie Studi di Storia Contemporanea $\beta$ www.diacronie.it}

Risorsa digitale indipendente a carattere storiografico. Uscita trimestrale. redazione.diacronie@hotmail.it

Comitato di redazione: Jacopo Bassi - Luca Bufarale - Elisa Grandi - Deborah Paci - Fausto Pietrancosta - Matteo Tomasoni - Luca Zuccolo

Diritti: gli articoli di Diacronie. Studi di Storia Contemporanea sono pubblicati sotto licenza Creative Commons 2.5 . Possono essere riprodotti a patto di non modificarne i contenuti e di non usarli per fini commerciali. La citazione di estratti è comunque sempre autorizzata, nei limiti previsti dalla legge. 